\title{
Entretien avec Jean-Yves Ruf, Éric Ruf et Loïc Corbery
}

Jean-Yves Ruf, Éric Ruf, Loïc Corbery, Leïla Adham et Jean-Michel Déprats

Christophe Hausermann (éd.)

\section{(2) OpenEdition}

Journals

Édition électronique

URL : http://journals.openedition.org/shakespeare/2840

DOI : $10.4000 /$ shakespeare.2840

ISSN : 2271-6424

Éditeur

Société Française Shakespeare

Édition imprimée

Date de publication : 1 mai 2014

Pagination : 179-192

\section{Référence électronique}

Jean-Yves Ruf, Éric Ruf, Loïc Corbery, Leilla Adham et Jean-Michel Déprats, «Entretien avec Jean-Yves Ruf, Éric Ruf et Loïc Corbery ", Actes des congrès de la Société française Shakespeare [En ligne],

31 | 2014, mis en ligne le 01 mai 2014, consulté le 01 mai 2019. URL : http://journals.openedition.org/ shakespeare/2840; DOI : 10.4000/shakespeare.2840 


\title{
EnTRETIEN AVEC JEAN-YVES RUf, Éric RUF ET LOÏC CORBERY
}

\author{
conduit par Leïla ADHAM et Jean-Michel DEPRATS
}

Jean-Michel Déprats : Bienvenue à nos invités Jean-Yves Ruf, Éric Ruf, et Loïc Corbery, respectivement metteur en scène, scénographe et comédien (Ulysse), et comédien interprète (Ajax) du Troilus et Cressida qui se donne à la Comédie-Française depuis le 26 janvier 2013.

Troilus et Cressida, qui reprend le thème de la guerre de Troie, est une pièce singulière, inclassable, considérée par la critique anglaise comme une des problem plays, pièces dont l'interprétation est problématique. Elle ne figure pas dans le «Catalogue » (table des matières) de l'in-folio de 1623 où elle est intercalée entre les Histoires et les Tragédies, juste après Henri VIII et immédiatement avant Coriolan. De fait, Troilus et Cressida transcende les divisions en genre. Il s'agit d'une pièce complexe, rarement montée en France depuis 1974, année où Stuart Seide l'a mise en scène avec Benoît Régent, Laurence Roy, Yves Gourvil, Wladimir Yordanoff et d'autres au Théâtre de l'École Normale Supérieure puis au Théâtre National de Chaillot. Declan Donnellan, en 2008, l'a également montée aux Gémeaux. Aujourd'hui, Jean-Yves Ruf relève le défi, et la pièce se joue en alternance jusqu'au 5 mai dans la salle Richelieu.

C'est sur le plan linguistique une ouvre d'une grande complexité, fourmillant de mots qui sont, sinon des hapax ou des néologismes, des termes singuliers, étranges, des vocables d'inspiration latine difficiles à déchiffrer tels vambrace, tortive, protractive, persistive, insisture, fixure, flexure, rejoindure etc... Il est très difficile de traduire ce langage d'allure érudite et aucune traduction nouvelle de cette pièce ne peut être une tentative de clarification maximale. Il faut par conséquent saluer le travail d'André Markowicz, qui l'a traduite ${ }^{1}$ sans complaisance, mais a écrit un texte dynamique et porteur de jeu.

\footnotetext{
1 William Shakespeare, Troülus et Cressida, traduit par André Markowicz, Préface de Margaret Jones-Davies, Paris, Les Solitaires Intempestifs, 2006.
} 
La première qualité du spectacle, à mon sens, est l'homogénéité de la troupe qui joue la pièce ${ }^{2}$ en ayant de toute évidence la volonté de la rendre aussi accessible que possible au public contemporain.

Leila Adham : Il est temps de présenter nos invités. Jean-Yves Ruf est metteur en scène et auteur. Il a intégré l'Unité nomade du Conservatoire de Paris après avoir suivi une formation de comédien au TNS. Il a mis en scène une vingtaine de spectacles, dont le très touchant Erwan et les oiseaux et Unplusun, deux pièces dont il est l'auteur, Silures d'après Coleridge, La passion selon Jean de Tarentino, La lettre au père de Kafka. Pour ce qui est de Shakespeare, il a monté Mesure pour mesure et Comme il vous plaira.

Éric Ruf, sociétaire de la Comédie-Française depuis 1998, est à la fois acteur, metteur en scène et scénographe. Il a joué, pour ne citer qu'eux, sous la direction de Patrice Chéreau, de Catherine Hiegel, d'Yves Beaunesne et de Jean-Yves Ruf. Il a travaillé comme scénographe avec Denis Podalydès et Frédéric Bélier-Garcia et a mis en scène Denis Podalydès dans Le Cas Jekyll à Chaillot. Plus récemment, il a mis en scène Peer Gynt d'Ibsen au Grand Palais.

Jean-Michel Déprats : C'était là un acte très courageux car la mise en scène de Patrice Chéreau était inoubliable. Pourtant, vous avez mis ce Peer Gynt en scène de manière tout à fait neuve et avec beaucoup d'humour.

Éric Ruf : On a le droit de réessayer tous les trente ans !

Jean-Michel Déprats : La distribution, avec notamment Hervé Pierre dans le rôle titre et Catherine Samie dans le rôle de Åse, la mère de Peer Gynt, était particulièrement brillante.

Leila Adham : Je reviens à Troilus et Cressida et j'en termine en vous présentant Loïc Corbery, acteur et sociétaire de la Comédie-Française

\footnotetext{
2 La distribution du spectacle comprend Yves Gasc, Michel Favory, Eric Ruf, Bruno Raffaelli, Laurent Natrella, Michel Vuillermoz, Christian Gonon, Loïc Corbery, Stéphane Varupenne, Gilles David, Georgia Scalliet, Jérémy Lopez, Louis Arene, Sébastien Pouderoux, Akli Menni, Laurent Cogez, Carine Goron, Lucas Hérault, Blaise Pettebone, Nelly Pulicani et Maxime Taffanel.
} 
depuis 2010 3 . Il a cette saison joué avec brio le rôle de Dom Juan sous la direction de Jean-Pierre Vincent. Auparavant, Loïc Corbery a travaillé également avec Catherine Hiegel, Yves Beaunesne, Alfredo Arias, et Denis Podalydès, entre autres.

Jean-Michel Déprats : Jean-Yves Ruf, pourquoi choisir de mettre en scène une pièce aussi difficile ? Comment arrive-t-on à une ligne dramaturgique lisible à partir d'une œuvre aussi compliquée ?

Jean-Yves Ruf : J'ai monté trois Shakespeare. Ce sont toutes des pièces à problème. J'ai monté Comme il vous plaira...qui n'est pas à proprement parler une problem play mais qui est complexe. J'ai aussi monté Mesure pour mesure et donc, aujourd'hui, Troilus et Cressida. J'ai un goût certain pour les pièces monstres dans leur forme. Dans Troilus et Cressida il y a une hétérogénéité au niveau de l'écriture : des vers très nobles et des vers de mirliton ; justement, j'aime ce collage, ce mélange-là. Et mon enfance a été marquée par l'œuvre d'Homère. Cela m'amusait de voir à quel point Shakespeare retournait Homère et faisait de ses héros des antihéros, de pauvres petits hommes qui n'y arrivent pas. La guerre homérique est, dans la pièce de Shakespeare, une guerre qui n'avance pas, et où la noirceur humaine se révèle ! J'ai eu envie de proposer ce projet à Muriel Mayette 4 , qui a accepté. Je l'ai proposé en me disant que, vu la difficulté du texte, ce n'était pas plus mal d'avoir des comédiens qui savaient déjà ce qu'est un texte. Je ne le regrette pas. Avec cette troupe-là, par rapport à une troupe plus jeune qui serait sortie de l'école, je crois que j’ai gagné dix jours ! Dès les premières répétitions, les comédiens connaissaient leur texte, la conduite de la pensée était déjà là. Et j'ai vite compris qu'il ne faut pas vouloir faire le malin avec une pièce pareille. Si on l'entend, si on la comprend, c'est déjà une victoire. Il ne faut pas avoir d'emblée un parti pris, un parti pris très contemporain par exemple. Ce qui nous a demandé le plus d'efforts, c'est le gros travail de déchiffrage du texte, un travail de débroussaillage en quelque sorte. Il y a des scènes qui nous ont posé des problèmes très longtemps, jusqu'à la dernière semaine.

3 En 2011, il a joué dans Les Joyeuses Commères de Windsor de Shakespeare, On ne badine pas avec l'amour de Musset et L'Avare de Molière, et en 2012, du même Molière, dans Le malade imaginaire et Dom Juan ou le festin de pierre.

4 Administratrice générale de la Comédie-Française depuis le 4 août 2006. 
Jean-Michel Déprats : Avez-vous effectué des coupes avant l'engagement du travail scénique?

Jean-Yves Ruf : Oui.

Jean-Michel Déprats : Une notamment concernant le rôle d'Hélène. Pourquoi la pauvre Hélène passe-t-elle à la trappe ?

Jean-Yves Ruf : Parce qu'elle n'apparaît que dans une seule scène. Et c'est vrai qu'une coupe est toujours une trahison. Mais il fallait que je coupe car une pièce à la Comédie-Française doit durer trois heures avec entracte. Or, on voit Hélène dans une scène qui ne semble pas indispensable, même s'il faut se méfier, car, dans Shakespeare, tout est utile.

Éric Ruf : Il faut dire que, dans la pièce, Hélène a droit à une demi-page au cours du deuxième acte (II.ii) pour que l'on comprenne pourquoi on se bat pour elle depuis dix ans !

Jean-Yves Ruf : À l'acte III, scène i, Pandare vient demander à Hélène et Pâris de bien vouloir excuser Troilus pour le dîner à la table de Priam ; il y a un jeu assez drôle entre Pandare et le serviteur, mais il a bien fallu couper. Et cela m'amusait qu'il n'y ait pas d'Hélène du tout dans la pièce. Troilus et Cressida est une pièce difficile parce qu'elle donne l'impression d'avoir plusieurs centres. C'est une pièce sur la trahison, et d'ailleurs, Shakespeare lui-même trahit tout le temps son propre projet. On attend de l'action, une guerre, et il n'y en a jamais. Tout est construit pour nous conduire à ce combat entre Ajax et Hector et ce combat est un pétard mouillé ! On attend une grande histoire d'amour, mais l'histoire d'amour s'arrête soudainement. Cette pièce peut donc paraître trompeuse, mais moi, je la trouve noire et jubilatoire.

Jean-Michel Déprats : À propos de trahison, je voudrais saluer la façon très convaincante dont vous traitez la supposée trahison de Cressida. Vous montrez bien que Cressida n'est pas infidèle parce qu'elle choisirait de l'être ! Elle est infidèle car elle n'a pas la liberté d'être fidèle. Elle est contrainte à l'infidélité quand elle est échangée contre un 
prisonnier troyen, elle choisit d'appartenir à un seul homme (Diomède) pour ne pas tomber dans le statut d'objet sexuel de toute l'armée. C'est une scène d'une grande résonance contemporaine et Georgia Scalliet est remarquable dans le rôle de Cressida. Elle nous livre une analyse sociale du statut de la femme dans un contexte guerrier.

Jean-Yves Ruf : Le rôle de Cressida porte en germe un grand personnage. Elle aurait pu être Juliette ! Lorsqu'elle apprend que les Troyens vont l'échanger contre un prisonnier troyen, elle dit qu'elle renie son père, qu'elle renie sa lignée, qu'elle préfère mourir plutôt que de quitter Troilus, alors que Troilus, lui, obéit à son père. Et d'ailleurs, à propos de Cressida, je crois que la vraie violence de la pièce, c'est qu'elle est absente du cinquième acte.

Leila Adham : Évoquons à présent le traitement de l'espace et la scénographie. J'ai été frappée par le caractère évolutif du dispositif scénique. Au début, tout se joue pratiquement sur une étroite bande latérale de scène, au plus près des spectateurs, et le dispositif gagne en profondeur au fur et à mesure que la pièce se déroule. Au dernier acte, ce qui est très beau, c'est que vous arrachez le mur du lointain, laissant ainsi apparaître la structure de l'arrière-scène.

Éric Ruf : La pièce est écrite de manière bipolaire. Elle se caractérise par d'incessants allers et retours entre les Grecs et les Troyens. Il fallait que l'on suggère deux espaces caractérisés mais sans lasser le spectateur, un peu à la manière dont on tournerait la page d'un livre. Il fallait aussi trouver une solution concrète, afin que le public sache qui est qui, qui joue dans quelle équipe, cela d'autant plus qu'il y a des transfuges dans Troilus et Cressida ! Nous avons donc décidé de mettre les Troyens à l'abri d'un mur, dans des boiseries, dans quelque chose de manufacturé. Autrement dit, dans un espace confiné. Les Grecs, eux, se retrouvent sur une plage absurde, entre Troie et les bateaux. Ils ont même pris les voiles de leurs navires pour se protéger du soleil ! Ils se trouvent dans un camp désinvesti. Les deux espaces sont donc très distincts. À la fin de la pièce, nous faisons tomber les mâts, nous faisons tomber les toiles, nous révélons la cage de scène. 
Jean-Michel Déprats : Ce dispositif fonctionne donc comme une forme de théâtre dans le théâtre...

Éric Ruf : Oui, et cela reflète la volonté de Jean-Yves. Il souhaitait aussi avoir des escaliers sur scène. On se pose souvent la question : que faire du corps dans les scènes immobiles, les scènes de pensée ? Le corps doitil être assis à ce moment-là ? Doit-il marcher ? Le fait d'avoir des degrés inscrit le corps dans l'espace. Nous avons donc créé des gradins. Je trouvais intéressant de faire évoluer le corps des acteurs sur ces escaliers.

Leila Adham : Avez-vous également utilisé ces gradins afin de mettre en image l'enjeu du regard dans la pièce ? Chacun se regarde comme au spectacle dans la pièce. Il y a un très fort effet de miroir entre la scène et la salle.

Éric Ruf : Tout à fait. Il fallait proposer des " chemins d'écoute », comme dit Jean-Yves. Il ne faut jamais se désinvestir dans une pièce comme celle-là. Il est donc bon que les acteurs puissent se placer dans ces gradins. La guerre se regarde, elle est devenue commune, journalière, comme un match ou une compétition sportive à laquelle on assiste. Les gradins mettent en lumière l'aspect désarmé des personnages. Les deux équipes ont ceci en commun que la guerre est vidée de sens. Les escaliers sont des escaliers transfuges, et cela nous permet de montrer que tous les personnages se demandent ce qu'ils font là.

Jean-Michel Déprats : Les lecteurs et les spectateurs de Troilus et Cressida ont toujours du mal à rentrer dans le premier acte de la pièce. C'est un acte durant lequel les discours s'accumulent. Le dramatisme, la dynamique scénique ne s'affirment qu'après l'acte I. Comment avezvous géré cette difficulté ?

Jean-Yves Ruf : Tous les premiers actes de toutes les pièces sont difficiles car il y a toujours des scènes d'exposition à gérer. En fait, il y a deux grandes scènes qui sont peu évidentes, l'une avec les Troyens, l'autre avec les Grecs. Volontairement je les ai peu coupées, car c'est tout le charme de Troilus et Cressida que de proposer une alternance de 
scènes de pensée et de scènes de clowns. Chez les Grecs, il y a la scène de clowns entre Thersite et Ajax, par exemple. Nous avons décidé d'utiliser la salle. Ulysse, joué par Éric, privilégie l'interaction avec la salle. Sinon, on ne l'écouterait pas aussi bien. Il partage sa pensée avec le public, qui doit, c'est vrai, être concentré. Mais je crois qu'il faut assumer la pièce de cette manière.

Jean-Michel Déprats : Parler de ces scènes discursives offre en effet une transition opportune pour évoquer le rôle d’Ulysse. Même les gens qui connaissent mal la pièce connaissent la tirade sur la hiérarchie (scène III de l'acte I), morceau de bravoure censé naguère refléter la pensée du dramaturge, illustrant l'importance de l'ordre. C'était du moins l'interprétation qu'en faisait E.M.W. Tillyard. Est-ce que pour vous, Éric Ruf, interprète du rôle d'Ulysse, ce discours a une valeur de référence ? Ou êtes-vous plutôt dans la recherche d'une voix singulière à l'intérieur d'un chœur?

Éric Ruf : Je savais que cette tirade était connue des universitaires, mais ce qui est décisif, c'est le passage au plateau. On a beau décider de grandes choses sur le texte quand on travaille à la table, c'est sur le plateau que tout se vérifie ou ne se vérifie pas, et dans ce lieu-là, personne ne possède de guide. Là, quelque chose se joue. Bien sûr, cette tirade fait partie des morceaux de bravoure. Par le biais de ce texte-là, le rapport avec la salle a été trouvé. La salle écoute quelque chose qui représente l'ordre du monde. Lorsque nous jouons la pièce, tout à coup, nous sentons que les gens écoutent, et cette écoute n'est d'ailleurs pas forcément de l'ordre de la compréhension. La tirade d'Ulysse suit immédiatement une scène de clown avec Pandare, et du coup, les gens sont surpris, ils sont saisis. Cette tirade réussit avec de l'humour et fonctionne beaucoup moins bien lorsque l'on y injecte de l'héroïsme. Pour interpréter cette matière verbale, il faut donc trouver une vitesse de croisière. D'un côté, on ne peut pas aller à un rythme trop lent, si l'on détaille trop les choses, on complexifie la pièce. D'un autre côté, si l'on va trop vite, si la tirade ne devient qu'une logorrhée brillante, les gens abandonnent aussi. Il faut par conséquent trouver un entre-deux, ce qui n'est pas simple. Heureusement, l'investissement du souffle de l'acteur pallie les choses trop compliquées. Et il existe au moins une structure, contre laquelle il faut se battre, d'ailleurs. Cette structure formelle 
ressort particulièrement de la traduction d'André Markowicz, qui a voulu tout traduire en décasyllabes. À ce moment-là, je travaillais moimême le personnage de Pyrrhus dans Andromaque! Il y avait aussi une vraie structure chez Racine. Les vers de Shakespeare et le vers de Racine diffèrent, bien sûr, mais à chaque fois, on retrouve du sens qui s'appuie sur une forme.

Jean-Michel Déprats : Comment définiriez-vous précisément le personnage d'Ulysse? Quelle est sa fonction sur l'échiquier politique des Grecs ? C'est un personnage structurant dans la pièce ?

Éric Ruf : Oui, parce qu'il est metteur en scène. On ne sait pas encore qu'ils vont mettre dix ans à rentrer, mais Ulysse est l'un de ceux qui a le plus envie de pénétrer dans le camp des Troyens, en privilégiant la real politik. Quand il peut convaincre les hommes de se battre et faire naître les détestations, il le fait.

Jean-Michel Déprats : Pour vous, ce n'est donc pas principalement un théoricien, un penseur, c'est un homme d'action.

Éric Ruf : Absolument. Il a toujours trois coups d'avance sur les autres.

Jean-Michel Déprats : Il est extrêmement critique envers son camp.

Éric Ruf : Oui, mais il le réveille, il le stimule aussi ! Il prononce de grandes vérités douloureuses. Il ne cesse de dire aux uns et aux autres ce qui est en train de se passer. Le premier discours inaugural d'Ulysse, c'est cela. Alors que l'on s'efforce de nuancer les difficultés, que Nestor utilise des images mythologiques, lui affirme très franchement que les Grecs sont en train de perdre parce qu'une personne reste sous sa tente et que rien ne pourra avancer. Encore une fois, dans la pièce, Ulysse est un metteur en scène qui s'appuie sur le savoir de Nestor, la crédulité d'Agamemnon et l'immense naïveté d'Ajax pour élaborer ses plans.

Loïc Corbery : Justement, je joue Ajax et je rentre juste après ce discours d'Ulysse sur la hiérarchie, en compagnie de Thersite, pour détendre un peu l'atmosphère. Avant cela, je suis en coulisses, à l'écoute de la salle et du plateau. Et je me rends bien compte, depuis les coulisses, 
qu'Éric prend complètement en charge le discours d'Ulysse. Certes, j'entends Ulysse qui s'adresse aux Grecs, mais j'entends aussi Éric parler de l'institution que peut être notre théâtre, qui est aussi une grosse machine ! Tout cela pour dire que le discours d'Ulysse n'est pas juste un discours inscrit dans l'Histoire. C'est aussi Shakespeare qui s'adresse à la salle, en 2013. Et c'est Éric qui dit qu'une hiérarchie est méprisée et qu'une entreprise est malade quand on agit de façon inconsidérée au niveau du pouvoir. Ce n'est pas rien à entendre. La personne qui prend en charge la parole d'Ulysse déteint sur cette parole. Toute tirade peut donc changer de couleur au théâtre, et pour nous en coulisses et pour les spectateurs dans la salle.

Jean-Michel Déprats : Et, dans ton interprétation, qui est véritablement Ajax dans la pièce?

Loïc Corbery : Je suis l'un de ceux qui parlent le moins.

Jean-Michel Déprats : Mais que l'on voit le plus.

Loïc Corbery : Ajax est souvent décrit, il est souvent présent. On le raconte plus qu'il ne se raconte. Dans cette aventure qu'est la représentation d'une pièce comme Troilus et Cressida, le grand plaisir, c'est d'ailleurs d'être en « bande ».

Jean-Michel Déprats : Je l'avais souligné d'entrée de jeu comme un des liens forts du spectacle. Cette dimension collective est très présente dans la mise en scène. La Comédie-Française, ce n'est pas simplement l'addition de talents individuels exceptionnels. Il y a un travail d'équipe.

Loïc Corbery : Oui, la dimension collective est importante, et c'est un bonheur de travailler ensemble. La pièce a des enjeux merveilleux sur la langue, sur la matière verbale elle-même. Un acteur doit explorer cette matière, la manger, doit suer et transpirer dessus. Je parle en général, car moi, je n'ai que de la prose, une phrase, puis quatre vers de temps à autre!

Jean-Michel Déprats : Mais tu viens de jouer Dom Juan ! 
Loïc Corbery : Oui, par conséquent, jouer Ajax, c'est un grand moment de repos ! Je dois dire que j'étais un peu frustré au départ. Mais le dialogue incessant du corps et de l'esprit que l'on trouve dans la pièce de Shakespeare est formidable. Moi j'avais surtout à donner corps à mon personnage.

Leila Adham : Nous n'avons pas encore parlé du plaisir du spectateur. Il y a une réelle sensualité dans le jeu d'acteurs, avez-vous travaillé cette sensualité, avez-vous cherché à la développer ?

Jean-Yves Ruf : Je prends le thème comme se rapportant en particulier aux rapports ambivalents des ennemis les uns vis-à-vis des autres. L'un des plus beaux thèmes abordés par la pièce, c'est ce mélange d'admiration et de haine entre ennemis. Les personnages se déplacent en délégations, leurs sentiments sont très partagés. On se nargue, on détaille le corps de l'autre. La scène où l'on voit ensemble les deux grands héros que sont Hector et Achille est très belle, très sensuelle.

Jean-Michel Déprats : C'était déjà le cas chez Homère d'ailleurs, qui insiste sur l'amitié profonde, l'estime profonde que se vouent des ennemis. Et c'est fréquent chez Shakespeare. On pense, par exemple, à l'érotisation des rapports entre Aufidius et Coriolan dans Coriolan.

Leila Adham : Si toutefois cela se travaille, comment avez-vous travaillé cette donnée?

Jean-Yves Ruf: Il ne faut pas avoir d'a priori sur une bande, une meute d'hommes. Nous avons aussi essayé de comprendre les différences de chacun.

Jean-Michel Déprats : Entre parenthèses, dans la scène où Cressida est embrassée, écrasée, happée par l'un puis par l'autre, comme dans une «tournante » métaphorique, une meute d'hommes, on voit ce que ce peut être!

Jean-Yves Ruf : Tout à fait. Shakespeare ne donne que des indices sur cette meute, et c'est en travaillant que l'on comprend les choses. Ce qui est intéressant, ce sont par exemple les rapports entre Patrocle, Thersite 
et Achille. Ce trio s'est éclairé au fur et à mesure de notre travail, qui a mis au jour la rivalité de Patrocle et de Thersite face à Achille. L'amitié des concurrents, comme dirait Deleuze, est l'un des thèmes très forts de cette pièce.

Jean-Michel Déprats : Le moment est peut-être venu de passer la parole à la salle.

Line Cottegnies : Avez-vous travaillé avec le traducteur avant les répétitions, et/ou pendant les répétitions?

Jean-Yves Ruf : Nous avons beaucoup travaillé avec André Markowicz. J'avais déjà travaillé avec lui pour Comme il vous plaira. Il $\mathrm{y}$ avait des choses, dans la pièce, que je ne comprenais pas, je lui ai envoyé énormément de notes et de questions, et il accepté d’y répondre. Nous avons négocié chaque chose, nous sommes rentrés dans les structures de la pièce, et j'ai vu grâce à lui qu'un seul mot peut irradier toute la pièce. Ensemble, nous avons conçu quatre ou cinq moutures différentes de la pièce. Et nous avons consacré trois ou quatre jours à la lecture de la pièce en sa présence avec les comédiens. Ensuite, nous avons continué à communiquer avec André Markowicz, à distance cette fois. Au cours des répétitions, nous l'avons abreuvé de mails ! Nous avions aussi le texte anglais à notre disposition pour mieux comprendre la pièce, car nous avons dans la troupe quelques anglicistes. André nous disait « je publie ce passage comme ça, mais sur le plateau, on peut faire autrement ! » Nous avons négocié jusqu'au bout. La pièce est si complexe !

Loïc Corbery : André nous donnait des réponses sur le sens, mais il avait aussi des clés organiques à nous donner pour nous aider à accéder à la pièce. Nous avions des contraintes de travail passionnantes à explorer. Un souffle pour une pensée. Il y a des règles... que l'on peut aussi briser de temps à autre, bien sûr !

Jean-Yves Ruf : André a une obsession : il faut s'arrêter au point, pas avant. 
Loïc Corbery : Les obsessions sont parfois démesurées mais ces contraintes, ces pistes de travail permettent de trouver un bon équilibre, un bon rythme, d'être dans le souffle mais pas dans un rythme trop rapide, pour que la pièce devienne intelligible sur un plan cérébral et organique.

Jean-Yves Ruf : Si on agrège des numéros d'acteur, on n'entend plus le poème. Il fallait trouver des règles communes, que chacun pouvait s'approprier ensuite à sa façon. Il y avait une manière de prendre la langue qui était collective.

Éric Ruf : Dans les répétitions, on ne sait pas toujours quoi faire avec le texte. Parfois, quand on joue Molière ou Racine, on ne se pose plus de questions et c'est souvent une erreur. Mais là, comment faire avec cette matière tous ensemble ? La troupe est notre fil d'Ariane. Il faut trouver, tous ensemble, comment s'y prendre.

Loïc Corbery : Et puis il y a aussi une sensualité propre à la langue de la pièce, je veux parler de la sensualité organique des mots. Quand j'écoute les autres, j'entends cette sensualité des mots.

Jean-Michel Déprats : Pour le traducteur, il y a une tension particulière à cette pièce, une tension extrême entre fidélité à un matériau linguistiquement compliqué et, d'autre part, nécessité de dynamisme scénique et d'intelligibilité. D'un côté, la pièce, complexe, comprend de nombreux néologismes, des termes singuliers ne référant à aucun vocable repérable ou à aucune notion connue. De l'autre, elle est destinée à être jouée et comprise. Il faut donc à la fois préserver la complexité du texte et favoriser une certaine fluidité pour que le public comprenne ce qui est en jeu.

Margaret Jones-Davies : En tant que spectatrice, déçue par de nombreuses mises en scène antérieures de Troilus et Cressida, je me préparais mentalement à partir à l'entracte ! Mais tout à coup Pandare arrive, il nous fait rire, il nous intègre dans son monologue. Nous sommes dans la comédie immédiatement. Et c'est gagné. 
Éric Ruf : Nous avons la même peur au début du spectacle ! Nous sommes tous en coulisses à ce moment-là, et nous demandons à l'acteur de bien élever la voix pour qu'il s'adresse aux spectateurs. Et nous attendons et écoutons la réponse du public...

Margaret Jones-Davies : Je suis très sensible aux décors. Je n'aime pas les décors qui ne représentent rien, qui nient le besoin du spectateur de se projeter, de rêver. Dans le vôtre, on voit la mer, il y a immédiatement un imaginaire! On a besoin de cela. Dans ce décor-là, j'ai lu l'amphithéâtre comme une illustration de la hiérarchie, du degree exalté par le discours d'Ulysse. Le sens de la pièce apparait dans le décor et cela a emporté mon adhésion. Ce grand mur par-dessus lequel il faut se pencher pour voir l'autre monde évoque bien le thème de la perception. La pièce est là, le décor fait bien ressortir la modernité de l'œuvre. J'ai enfin beaucoup aimé le personnage d'Ajax. Il m’a très étrangement rappelé Hamlet car Hamlet est fou et Ajax est le grand fou de Troilus et Cressida !

Isabelle Schwartz-Gastine : En ce qui me concerne, j'ai perçu la recherche d'une esthétique japonisante ou orientale dans la mise en scène, présente notamment dans les gestes rituels d'Achille. Est-ce que je me trompe?

Jean-Yves Ruf : Non. Ce sont surtout les costumes qui vous ont donné cette impression. Avec la costumière, nous avons regardé des images de samouraïs. Le maquillage participe également de cette esthétique, de façon discrète, surtout pour le groupe des Troyens. Le prince troyen Hector a une pensée complexe, il pense que la guerre est injuste et qu'il faudrait rendre Hélène mais il ne va pas plus loin que cela. Il ne raisonne qu'en termes de vertu et de hauteur d'âme. Et c'est finalement Ulysse qui gagne.

Éric Ruf : Cette composante japonisante est aussi liée à la difficulté de représenter les combats au théâtre. Il est risqué de représenter la guerre et la martialité au théâtre. Les épées, les lances et les boucliers, sur scène, cela donne très facilement un effet " playmobil »! Dès que l'on essaye de traiter sérieusement les affrontements guerriers, on redouble le ridicule de la mise en scène. Nous sommes donc allés vers le côté 
japonisant : un seul coup, plutôt que toute une série de figures non crédibles !

Jean-Michel Déprats : Cette difficulté que vous pointez (à représenter la guerre et les combats au théâtre), Shakespeare en faisait déjà état dans Henry $V$ où le Chœur de l'Acte I, sollicitant la collaboration du public, dit aux spectateurs : «Suppléez à nos imperfections par vos pensées /Divisez chaque soldat en mille, / Et créez une armée imaginaire » 5 et où le Chœur de l'Acte IV évoque « quatre ou cinq épées minables et ridicules /Maladroitement croisées dans une rixe dérisoire »...Le temps est hélas venu de clore notre entretien et de vous remercier tous trois très, très chaleureusement d'avoir accepté de participer à cette rencontre.

Propos recueillis par Sophie Chiari et mis en forme par Jean-Michel Déprats

\footnotetext{
5 William Shakespeare, La Vie d'Henry V, Traduction par Jean-Michel Déprats, in Histoires, II, Euvres Complètes, IV, Jean-Michel Déprats et Gisèle Venet éds, Paris, Gallimard, Bibliothèque de la Pléiade, 2008, Prologue, p. 731
} 